\title{
ANALISE DO IMPACTO DO ICQ E CC SOBRE O RISCO CARDÍACO EM PESSOAS COM BAIXOS NÍVEIS DE ATIVIDADE FÍSICA
}

Romulo Jose Dantas Medeiros

Maria do Socorro Cirilo de Sousa

Giulliana Nobrega Cantisani

Anderson Carlos Lourenço de Lima

Mônica da Silveira Torres

João Marcos Ferreira de Lima Silva

Luciano Meireles de Pontes

Luciano Carvalho de Oliveira

Gilmario Ricarte Batista

\section{Resumo}

Objetivo: Analisar o impacto do índice cintura/quadril (ICQ) e circunferência da cintura (CC) sobre o risco cardíaco (RC). Material e Métodos: Estudo descritivo, transversal e quantitative, composto por uma amostra selecionada voluntariamente, na adesão as academias de ginástica de 75 mulheres (M) e 47 homens (H), media de 30,03+11,33 anos, medidos na antropometria em instrumentos calibrados. Utilizou-se SPSS 13,0 para descritiva e "t"de Student. Significancia 5\% . Resultados: 42,6\% e 23,4\% dos $\mathrm{H}$ apresentam-se na faixa de RC alto e muito alto para ICQ e 40,9\% e 18,2\%, risco aumentado e muito aumentado para o CC, respectivamente; $28,0 \%$ e $61,3 \%$ das $\mathrm{M}$ apresentam-se na faixa de risco alto e muito alto para ICQ e $59,1 \%$ e $30,6 \%$, risco aumentado e muito aumentado para o CC, respectivamente; $\mathrm{p}=0,000$, diferentes entre gêneros e ICQ e CC sao de independentes $(\mathrm{r}=0,460)$. Conclusao: O ICQ e CC representam impacto no risco cardíaco e ambos os gêneros estão propensos a desenvolver distúrbios cardiovasculares.

\section{Palavras-Chave}

Cintura/quadril; Circunferência de cintura e risco cardíaco

\section{IT ANALYZES OF THE IMPACT OF ICQ AND CC ON THE CARDIAC RISK IN PEOPLE WITH LOW LEVELS OF PHYSICAL ACTIVITY}

Romulo Jose Dantas Medeiros

Maria do Socorro Cirilo de Sousa

Giulliana Nobrega Cantisani

Anderson Carlos Lourenço de Lima

Mônica da Silveira Torres

João Marcos Ferreira de Lima Silva

Luciano Meireles de Pontes

Luciano Carvalho de Oliveira

Gilmario Ricarte Batista

\begin{abstract}
Objective: To analyze the impact of index waist/hip (IWH) and waist circumference on the cardiac risk (CR). Material and Methods: Descriptive, transversal and quantitative research, sample selected voluntarily, in the adhesion to the academies of gymnastics of realized with 47 men (M) and 77 women
\end{abstract}


(W), 30,03 + 11, 33years middle-aged, measured in the anthropometry in calibrated instruments. One used SPSS 13,0 for descriptive and "t" of Student. Significance 5\% Results: 42,6\% e 23,4\% of the M are in the high RC and very high RC zone to IWH, respectably, and 40,9\% e 18,2\%, increased risk and very increased risk to $\mathrm{CC}$, respectably; $28,0 \%$ e $61,3 \%$ of the $\mathrm{W}$ are in the high $\mathrm{CR}$ and very high $\mathrm{CR}$ zone to IWH, respectably e $59,1 \%$ e $30,6 \%$, increased risk and very increased risk to WC, respectably; $\mathrm{p}=0,000$ between sexs and IWH and WC are of low correlation, that is, independent $(r=0,460)$ Conclusion: IWH and WC represent impact in the cardiac risk and both the sorts and are inclined to develop cardiovascular riots.

\section{Key-Words}

Index waist/hip; Waist circumference and cardiac risk. 


\section{INTRODUÇÃO}

A sociedade moderna tem sido acometida por um numero progressivamente maior de pessoas que apresentam alguma disfunção cardiovascular, fazendo assim com que tais disfunções ou doenças sejam uma das principais causas de óbito na atualidade (BOTREL et al., 2000). Nas ultimas décadas, o surgimento dessas patologias relacionou-se, em um grau cada vez mais relevante e preocupante, com o estilo de vida adotado por diversas pessoas, que se tornou hipocinetico em conseqüência dos confortos proporcionados pela modernidade (NEGRÃO; BARRETTO, 2005).

Nesse contexto, Pontes et al. (2006) ao mencionar o relatório sobre a Saúde no Mundo de 2002, afirma que o desenvolvimento de patologias relacionadas a hipocinesia tem uma forte relação com determinados fatores, tais como: tabagismo, obesidade, hipertensão, entre outros. Pollock e Wilmore (1993), criteriosamente, evidenciam que as doenças cardiovasculares, também mencionadas como cronico-degenerativas não transmissíveis (DCNT), podem surgir a partir da exposição dos indivíduos a certos fatores, que neste, são classificados de acordo com o seu risco, são eles: fatores de risco primários (FRP) e secundários (FRS).

Presentemente, a hipertensão, o tabagismo e os altos níveis de colesterol sanguíneo foram identificados como FRP. Entre os FRS, estão incluídos aqueles que podem ser controlados ou alterados, ou seja, o estresse e desequilíbrio emocional, a obesidade, o diabetes e a inatividade física. Ha também os FRS que não são passiveis de alterações, que são: idade, sexo e hereditariedade. No âmbito dos fatores de risco secundários, encontra-se uma variável que estar em crescente evidencia e presença no cotidiano da população atualmente, que são os níveis altos de gordura corporal, estando esses diretamente vinculados a estilos de vida sedentários, alimentação hipercalórica rica em gorduras e pobre em fibras (KATHLEEN e SCOTT-STUMP, 2005).

Muitos estudos tem evidenciado que a gordura corporal, principalmente a localizada na região abdominal, apresenta um grande impacto sobre as doenças cardiovasculares por associar-se com grande freqüência a condições tais como dislipidemias, hipertensão arterial, resistência a insulina e diabetes, que por sua vez, favorecem a ocorrência de eventos cardiovasculares, particularmente os coronarianos (WORLD HEALTH ORGANIZATION, 1998; KANNEL et al., 2002). Em relação a avaliação da gordura abdominal, diversas técnicas podem ser utilizadas para predizer de maneira eficaz essa medida, no entanto, as mais utilizadas são aquelas que apresentam baixo custo de aplicação e oferecem Conexões: revista da Faculdade de Educação Física da UNICAMP, Campinas, v. 6, ed. especial, p. 184-196, jul. 2008.186 ISSN: $1983-9030$ 
confiabilidade nos seus resultados. Para as características citadas, as que melhor se enquadram são: circunferência da cintura (CC) e índice cintura/quadril (ICQ) (FERREIRA et al., 2006).

Petroski (1999), explica que o uso da antropometria e indicado pela Organização Mundial da Saúde (OMS), para a vigilância de fatores de risco nas doenças crônicas, e é recomendada na analise da relação dos parâmetros antropometricos, com desenlaces como a pressão arterial, em diferentes paises. Pereira et a/.(1999) afirmam que alem da massa corporal e estatura, devem ser medidos os perímetros da cintura e do quadril, pois o aumento da deposição de gordura abdominal pode ser um indicador sensível relacionado ao sobrepeso e suas conseqüências. As medidas antropometricas mais utilizadas são a medida da cintura (CC) e a relação cintura/quadril (RCQ) (CABERRA FILHO, 2001).

Todos esses parâmetros de adiposidade tem, individualmente, mostrado correlação significativa com hipertensão arterial, independentemente da origem étnica e condição socioeconômica dos indivíduos (PETROSKI, 1999). Ha varias outras medidas sugeridas, dentre elas o índice de conicidade (IC), que, segundo VALDEZ, tem potencial de prognostico, entre a distribuição de gordura e doença (HEYWARD, STOLARCZYK, 2000). O ICQ e a medida obtida através da divisão dos perímetros de cintura e quadril, e que de acordo com Pereira (1999), apresenta-se ideal quando os homens tem $<95$ e as mulheres $<80$. No caso da $\mathrm{CC}$, os pontos de corte utilizados para a classificação são definidos por Lean et al. (1995), que associam risco aumentado (nível 1) e risco muito aumentado (nível 2) aos seguintes valores: nível 1 para homens e mulheres $(\mathrm{CC}>94 \mathrm{~cm}$ e $\mathrm{CC}>80$, respectivamente) e nível 2 ara homens e mulheres ( $\mathrm{CC}>102 \mathrm{~cm}$ e $\mathrm{CC}>88$, respectivamente).

Diante do exposto, observa-se a necessidade de intervenção com o intuito de reduzir a propensão de desenvolver patologias de origem cardiovascular por parte da população adepta a atividade física. Logo, o presente estudo objetiva analisar o impacto do índice cintura / quadril e perímetro da cintura sobre o risco cardíaco em pessoas com baixos níveis de atividade física.

\section{METODOLOGIA}

\section{Características da Pesquisa}

A pesquisa caracterizou-se como descritiva do tipo transversal, de caráter quantitativo e utilizou-se do método estatístico para a analise e obtenção dos dados. Segundo RUDIO (2002), a pesquisa descritiva descreve através da observação características de um determinado fenômeno, no qual, através de parâmetros estatísticos, o método quantitativo o quantifica e analisa. e é do tipo transversal por que os 
dados são coletados em um único instante no tempo, obtendo assim, um recorte de informações momentâneo.

\section{POPULAÇÃO E AMOSTRA}

A população estudada foi de indivíduos iniciantes de atividade física. A amostra foi composta por 122 pessoas, que ingressaram em academias de ginástica, sendo 75 mulheres e 47 homens, estando os mesmos em uma faixa etária de 18 a 68 anos, com media de idade 30,03+11,33 anos, selecionados voluntariamente, de acordo com a adesão ao estabelecimento. Os critérios amostrais foram: estar iniciando um programa físico estruturado, ter 18 anos ou mais.

\section{INSTRUMENTOS PARA COLETA DE DADOS E VARIÁVEIS DO ESTUDO}

Foi administrado a todos os participantes um conjunto de instrumentos destinados a obter informações acerca das variáveis em analise neste estudo. As variáveis selecionadas para a pesquisa foram: idade (ID) em anos completes, massa corporal (MC) em kg e estatura (EST) em m, circunferência da cintura (CC) e índice cintura/quadril (ICQ) obtido através da divisão dos perímetros de cintura e quadril. Os instrumentos de medidas utilizados para coletas dos dados incluíram uma balança de alavanca com estadiometro modelo Filizola ${ }^{\circledR}$ com precisão de $100 \mathrm{~g}$ e $0,01 \mathrm{~cm}$, devidamente aferida e com selo Inmetro, compasso de dobras e fita antropometrica do fabricante Sanny com $150 \mathrm{~cm}$ de resolução.

\section{PROCEDIMENTOS PARA COLETA DE DADOS}

A coleta dos dados foi realizada por uma profissional de educação física durante os procedimentos de avaliação física efetuada nas intermediações da academia, em espaço destinado exclusivamente para tal fim. A coleta de dados teve duração de 8 meses, ou seja, de Janeiro a agosto de 2006. Durante todo o período de realização da coleta os participantes do estudo receberam esclarecimento sobre o objetivo da pesquisa, bem como sobre os procedimentos que seriam adotados para realização da mesma, consentindo em participar da pesquisa de forma voluntária. Todas as etapas da coleta de dados contemplaram as normas éticas do Conselho Nacional de Saúde para a realização de pesquisas com Seres Humanos, Lei 196/96 (BRASIL, 2002).

As medidas antropometricas foram todas realizadas com os sujeitos descalços, vestidos com roupas leves e sob a condição de repouso total.

Massa corporal: a medida foi realizada com o avaliado em pé, de costas para a escala, com os pés 
afastados no centro da plataforma.

Estatura: foi medida com o avaliado de pé, posição ereta, braços estendidos ao longo do corpo, pés unidos e cabeça paralela ao solo, estando o avaliado em apneia inspiratoria.

CC: para esta medida utilizou-se uma fita antropometrica, com o individuo em pé, abdomen relaxado, colocou-se a fita num piano horizontal, no ponto de menor circunferência, abaixo da ultima costela.

ICQ: obtido através da divisão do CC e circunferência de quadril.

\section{RISCO CARDÍACO (RC):}

Para classificação do RC relacionado ao CC, adotou-se ponto de corte nível 1 para homens e mulheres $(\mathrm{CC}>94 \mathrm{~cm}$ e $\mathrm{CC}>80$, respectivamente) e nível 2 ara homens e mulheres $(\mathrm{CC}>102 \mathrm{~cm}$ e $\mathrm{CC}>88$, respectivamente). Para a ICQ, os pontos de classificação de risco foram os estabelecidos por Bray e Gray (1988).

\section{PLANO ANALÍTICO}

Os procedimentos analíticos foram tabulados conforme os valores apresentados a partir da coleta de dados brutos e as proposições traçadas para o referido estudo. Os dados foram digitados e equacionados em planilha Microsoft Excel 2003 e os resultados transportados para o Statistical Package for the Social Science (SPSS) versão 13.0, para analise de estatística descritiva de media, desvio padrão, mínimo e máximo. Para a analise dos dados emparelhados foi utilizado o teste "t" de Student; para a observacao das associações entre variáveis foi feito uso do coeficiente de correlação " $r$ " de Pearson, sendo adotado ponto de corte de alto igual ou maior que 0,600 e nível de significância de 5\%. Os dados tabulados e analisados foram distribuídos em tabelas.

\section{RESULTADOS E DISCUSSÃO}

A tabela 1 caracteriza a amostra de valores das medidas estatísticas descritivas de media, desvio e o valor probabilidade $\mathrm{p}$ das variáveis antropométricas em ambos os sexos. Verificou-se que o grupo masculino apresentou valores de media superiores ao grupo feminino, com exceção da idade. Todas as variáveis descritas na respectiva tabela apresentam diferenças significativas quando relacionada aos gêneros $(\mathrm{p}<0,05)$. Considerando-se a media de idade de cada grupo (masculino e feminino), verifica-se que ambos são considerados de alto risco (QUEIROGA, 1998). 
Em um estudo realizado com 68 iniciantes de ambos os gêneros na pratica de exercícios físicos regulares em academias de ginástica, foram observados resultados semelhantes com o presente estudo, havendo nesse caso, uma maior predominância de valores indicativos de risco cardíaco no sexo masculino (OLIVEIRA FILHO et al., 2001). Pitanga e Lessa (2005) objetivando comparar vários indicadores antropometricos de obesidade e identificar dentre eles qual melhor discrimina o risco coronariano elevado (RCE) em estudo de corte transversal, com amostra composta por 968 adultos de 30 a 74 anos de idade, sendo $391(40,4 \%)$ do sexo masculino pelo índice de conicidade (índice C), razão circunferência cintura-quadril (RCCQ), circunferência de cintura(CC), encontraram que a maior área sob a curva ROC foi encontrada entre o índice Ce risco coronário elevado (RCE), em indivíduos do sexo masculino, 0,80 (0,74-0,85), diferindo significativamente dos demais indicadores de obesidade.

E em mulheres, a maior área sob a curva ROC encontrada foi de 0,76 (0,71-0,81), sendo iguais entre índice C, RCCQ e RCE concluindo-se que o índice C e RCCQ são os melhores indicadores de obesidade para discriminar RCE. A CC tem intermediário poder discriminatório e o IMC foi o indicador antropométrico de obesidade menos adequado para discriminar RCE. Estes dados sugerem que os indicadores de obesidade abdominal sao melhores para discriminar RCE que os indicadores de obesidade generalizada.

\section{Tabela 1: Media, desvio padrão e significancia " $t$ " de student das variáveis idade (anos), massa corporal (kg), estatura (m), ICQ e CC (cm) nos grupos masculino e feminino $(\mathbf{N}=122)$}

\begin{tabular}{cccccc}
\hline Variáveis & \multicolumn{2}{c}{$\begin{array}{c}\text { Masculino } \\
(\mathrm{n}=47)\end{array}$} & \multicolumn{2}{c}{$\begin{array}{c}\text { Feminino } \\
(\mathrm{n}=75)\end{array}$} & $\begin{array}{c}\text { Sig. } \\
\text { "t" de student }\end{array}$ \\
\hline & Média & $\begin{array}{c}\text { Desvio } \\
\text { padrão }\end{array}$ & Média & $\begin{array}{c}\text { Desvio } \\
\text { padrão }\end{array}$ & Valores \\
\cline { 2 - 5 } & 26,80 & $\pm 7,47$ & 31,85 & $\pm 12,67$ & $0,001^{*}$ \\
Idade (anos) & 78,17 & $\pm 14,41$ & 60,99 & $\pm 9,89$ & $0,000^{*}$ \\
$\begin{array}{c}\text { Massa Corporal } \\
\text { (kg) }\end{array}$ & 1,74 & $\pm 0,07$ & 1,60 & $\pm 0,65$ & $0,000^{*}$ \\
Estatura (m) & 0,91 & $\pm 0,54$ & 0,84 & $\pm 0,50$ & $0,000^{*}$ \\
ICQ & 92,87 & $\pm 10,89$ & 85,10 & $\pm 9,17$ & $0,000^{*}$ \\
CC (cm) & & & & &
\end{tabular}

$\mathrm{Na}$ Tabela 2 estão descritos os valores de classificação para ICQ relacionados ao risco cardíaco de ambos os sexos, evidenciando o numero, o percentual de freqüência por ICQ e sexo de indivíduos presentes nas determinadas zonas classificatórias. Encontrou-se que 10,6\% $(n=5)$ dos indivíduos do sexo masculino estão presentes faixa de risco baixo, representando assim $100 \%$ das pessoas componentes da 
respectiva classificação; 23 4\% $(n=11)$ dos homens e 10,7\% $(n=8)$ das mulheres, são classificadas como risco moderado, representando assim 15,6\% $(n=19)$ da população total; 42,6\% dos homens $(n=20)$ e $28,0 \%(n=21)$ das mulheres encontram-se na zona classificatória de risco alto, representando dessa forma, 33,6\% $(\mathrm{n}=41)$ do total de indivíduos e por final, $23,4 \%$ dos homens e $61,3 \%$ das mulheres encontram-se classificados na faixa de risco muito alto, representando através desses valores, 46,7\% do total de componentes da amostra.

Diante da descrição supracitada, verifica-se que ha uma maior percentual e numero de mulheres presentes na faixa de risco muito alto, enquanto os homens encontram-se em maior quantidade na faixa classificatória de risco alto. Nas demais faixas, ha predominância do sexo masculino. Os dados respectivos dados, não corroboram com os analisados por Amer et al. (2001), que evidenciaram não haver homens classificados na faixa de risco alto e muito alto, da mesma forma, que observaram haver um maior numero de mulheres na faixa classificatória de risco moderado $(50,09 \%)$, seguido por risco alto $(28,8 \%)$. Tais dados talvez não corroboram com os do presente estudo talvez por algumas características diferenciadas das amostras.

\section{Tabela 2: Impacto da Classificacao do ICQ sobre o risco cardíaco em ambos os Gêneros $\underline{(\mathrm{N}=122)}$}

\begin{tabular}{|c|c|c|c|c|}
\hline \multicolumn{2}{|c|}{ Vaníveis deClassificaçãodoICQ } & Masculino (n=47) & Feminino $(\mathrm{n}=75)$ & Total \\
\hline \multirow[t]{3}{*}{ Risco baixo } & Freqüência & 5 & 0 & 5 \\
\hline & \%FrecuânciaICQ & 100,0 & 0 & 100,0 \\
\hline & \% Frequiencia Sexo & 10,6 & 0 & 4,1 \\
\hline \multirow[t]{3}{*}{ Riscomoderado } & Freciência & 11 & 8 & 19 \\
\hline & \%FrequênciaICQ & 57,9 & 42,1 & 100,0 \\
\hline & \% Frequiencia Sexo & 23,4 & 10,7 & 15,6 \\
\hline \multirow[t]{3}{*}{ Risco alto } & Frequiência & 20 & 21 & 41 \\
\hline & \%FrequiênciaICQ & 48,8 & 51,2 & 100,0 \\
\hline & \% Frequiencia Sexo & 42,6 & 28,0 & 33,6 \\
\hline \multirow{3}{*}{$\begin{array}{c}\text { Risco muito } \\
\text { alto }\end{array}$} & Frequîncia & 11 & 46 & 57 \\
\hline & \%FrecuîncaiaICQ & 19,3 & 80,7 & 100,0 \\
\hline & \%Frecuiencia Sexo & 23,4 & 61,3 & 46,7 \\
\hline \multirow[t]{3}{*}{ Total } & Frequêancia & 47 & 75 & 122 \\
\hline & \%FrecuîncaiaICQ & 38,5 & 61,5 & 100,0 \\
\hline & \%Frecpiência Sexo & 100,0 & 100,0 & 100,0 \\
\hline
\end{tabular}

Na Tabela 3 descrevem-se os valores de classificação para CC relacionados ao risco cardíaco de ambos os 
sexos, destacando o numero, o percentual de freqüência por $\mathrm{CC}$ e sexo de indivíduos presentes nas determinadas faixas de classificação. Encontrou-se que 40,9\% $(\mathrm{n}=18)$ dos indivíduos do sexo masculino e 33,3\% (n=24) do sexo feminino, representando assim 36,2\% $(n=42)$ do total da população; 40,9\% $(\mathrm{n}=18)$ dos homens e 36,1\% $(\mathrm{n}=26)$ das mulheres são classificadas como risco moderado, representando assim 37,9\% $(n=44)$ de toda amostra e 18,2\% dos homens $(n=8)$ e 30,6\% ( $n=22)$ das mulheres encontram-se na zona classificatória de risco alto, representando dessa forma, 25,9\% $(n=30)$ do total de indivíduos.

Frente aos dados dispostos na tabela, verifica-se que ha uma maior percentual e numero de homens presentes na faixa de risco baixo e aumentado, no entanto, as mulheres destacam-se substancialmente quando se analisa a faixa de classificação de risco muito aumentado. Tais informações corroboram com estudos que evidenciam uma maior propensão de mulheres em serem obesas e consequentemente, apresentarem CC maiores (OLIVEIRA et al., 1998; CEDDIA, 1998). Em uma pesquisa realizada com 318 indivíduos, Sampaio e Figueiredo (2005), constataram que de acordo com a média de idade estabelecida para homens e mulheres, essas se classificam em um faixa de risco maior se comparadas aos homens

\section{Tabela 3: Impacto da Classificação da CC sobre o risco cardíaco em ambos os gêneros $\underline{(\mathrm{N}=122)}$}

\begin{tabular}{|c|c|c|c|c|}
\hline \multicolumn{2}{|c|}{ Varúveis de Classificaçãoda CC } & $\begin{array}{l}\text { Masculino } \\
\text { (11=47) }\end{array}$ & $\begin{array}{c}\text { Fermino } \\
(\mathrm{n}=75)\end{array}$ & Total \\
\hline \multirow[t]{3}{*}{ Risco barxo } & Freciuiencia & 18 & 24 & 42 \\
\hline & $\%$ Frequência CC & 42,9 & 57,1 & 100,0 \\
\hline & $\begin{array}{l}\text { \%Frequiencia } \\
\text { Sexo }\end{array}$ & 40,9 & 33,3 & 36,2 \\
\hline \multirow{3}{*}{$\begin{array}{l}\text { Risco aumentado } \\
\text { Nivel1 }\end{array}$} & Frequîncia & 18 & 26 & 44 \\
\hline & \%Frequiência $O C$ & 40,9 & 59,1 & 100,0 \\
\hline & $\begin{array}{l}\% \text { Frequiênca } \\
\text { Sexo }\end{array}$ & 40,9 & 36,1 & 37,9 \\
\hline \multirow{3}{*}{$\begin{array}{l}\text { Riscominto } \\
\text { almentado } \\
\text { Nivel2 }\end{array}$} & Frecüência & 8 & 22 & 30 \\
\hline & \%Frequência CC & 26,7 & 73,3 & 100,0 \\
\hline & $\begin{array}{c}\text { \%Frequiencia } \\
\text { Sexo }\end{array}$ & 18,2 & 30,6 & 25,9 \\
\hline \multirow[t]{3}{*}{ Total } & Frecuiência & 44 & 72 & 116 \\
\hline & $\%$ Frequência $O C$ & 37,9 & 62,1 & 100,0 \\
\hline & $\begin{array}{l}\text { \% Frequîênca } \\
\text { Sexo }\end{array}$ & 100,0 & 100,0 & 100,0 \\
\hline
\end{tabular}


No presente estudo foi possível observar que a correlação "r" de Pearson, indicou baixas correlações entre o ICQ e CC, tanto para homens quanta para mulheres, $r=0,460$, indicando que estes preditores são independentes, ou seja não se associam, e portanto, ambos devem ser

calculados em caso de estudos de pesquisa sobre o risco por perimetria, embora a CC seja eleita como preditora isolada mais eficiente do risco cardíaco pela obesidade centralizada. Segundo Powers e Howley (2000), indivíduos com grande circunferência de cintura associada a circunferência de quadril apresentam maior risco de doenças cardiovasculares (infarto e aterosclerose) e de morte súbita. Nesta pesquisa observou-se valores de media de CC de $85 \mathrm{~cm}$ para mulheres e $92 \mathrm{~cm}$ para homens, o que já representa valores propensos a desenvolverem patologias de ordem cardiovascular.

Andriola e Sousa (2007) objetivando estudar o risco cardíaco por referenda e perimetria (ICQ e CC) em uma amostra de 153 pessoas na cidade de João Pessoa, encontrou que em relação aos fatores de exposição ao risco cardíaco encontrou-se, 53,88\% por faixa etária, 77,5\% por excesso de peso, 61,9\% por hereditariedade, 9,9\% por tabagismo, 81,0\% por baixos níveis de exercício, $52,2 \%$ por hipercolesterolemia, $25,5 \%$ por PA acima de $120 \mathrm{mmHg}, 58,2 \%$ por Gênero feminino, apresentando assim, 55,6\% risco médio e 19,6\% risco alto, 58,7\% dos homens e 88,9\% das mulheres apresentaram ICQ elevados. Estes resultados corroboram com os encontrados nesta pesquisa, principalmente por se tratar de uma amostra da mesma população no que se refere ao risco pela CC e ICQ.

Percebe-se que a amostra apresenta fatores de risco coronariano na medida em que houve escores acima do nível médio, estes fatores devem ser controlados, pois podem levar a um risco iminente em caso de alguma mudança de habito e de vida ou quando não tratados. Pereira et al (1999), em um estudo no Rio de Janeiro, com 3.282 indivíduos com idade acima de 20, encontraram que os melhores pontos de corte para definir ICQ foram 0,90 e 0,80 para homens e mulheres respectivamente. Neste estudo os valores estão mais elevados, porem bem próximos para o grupo masculino, com ponto de corte 0,91 e para as mulheres 0,84. Diante do exposto, percebe-se como e alta a prevalência de pessoas de ambos os Gêneros (masculino e feminino), com grandes possibilidades de desenvolver distúrbios cardíacos, ao se analisar os valores de ICQ e CC. Dessa forma, e de extrema importância se estabelecer um programa de atividade física adequado, juntamente com uma dieta balanceada para que se 
possa proteger o organismo das pessoas contra o maior acumulo de gordura corporal, principalmente localizado na região abdominal, como enfatiza Guedes (1998).

\section{CONCLUSÃO}

Mediante as analises realizadas ao longo desta pesquisa e conseqüente avaliação dos valores das variáveis ICQ e CC, pode-se constatar que estas representam impacto sobre o risco cardíaco e ambos os Gêneros estão propensos a desenvolver distúrbios cardiovasculares, sendo que o grupo de mulheres apresentando maior prevalência. Foi possível também verificar a independência entre o ICQ e CC como preditores de risco cardíaco. Dessa forma, indica-se a necessidade do desenvolvimento de programa de exercício físico adequado, vinculado a uma estrutura alimentar apropriada, para que seja possível reduzir os fatores de risco cardíaco em praticantes iniciantes de atividade física em academias de ginástica.

\section{REFERÊNCIAS}

AMER, N. M.; SANCHES, D, M.; Solange, M. F. Índice de massa corporal e razão cintura/quadril de praticantes de atividade aeróbica moderada. Revista da Educação Física/UEM, Maringá. v. 12, n. 2, p. 97-103, 2001.

BRASIL, Ministério da Saúde. Manual operacional para comitês de ética em pesquisa. Brasília, DF., 2002

BRAY, G. A.; GRAY, D. S. Obesity. Pathogenesis.(pt.1) Western Journal of Medicine, v. 149, p. 429-441, 1988.

CEDDIA, R. B. Gordura corporal, exercícios e emagrecimento. Revista Sprint Magazine, Rio de Janeiro, n. 99, p. 10-20, 1998.

OLIVEIRA, M. R. M. et al. Fatores determinantes da obesidade em mulheres de 30 a 40 anos. Revista Nutrição em Pauta, São Paulo, n. 33, p.32,1998.

OLIVEIRA FILHO, A; SHJROMOTO, R. N. Efeitos do exercício físico regular sobre índices preditores de gordura corporal: índice de massa corporal, relação cintura-quadril e dobras cutâneas. Revista da Educação Física/UEM, Maringá, v. 12, n. 2, p. 105-112, 2001

FERREIRA, M. G.; et al. Acurácia da circunferência da cintura e da relação cintura/quadril como preditores de dislipidemias em estudo transversal de doadores de sangue de Cuiabá, Mato Grosso, Brasil. Caderno de Saúde Pública, Rio de Janeiro. v. 22. n. 2, p. 307-314, 2006.

GUEDES, D. P; GUEDES, J. E. R. P. Controle do peso corporal. Londrina: Midiograf, 1998. 
LEAN, M. E. J.; HAN, T. S.; MORRISON, C. E. Waist circumference as a measure for indicating need for weight mangement. BMJ v.31, n. 2, p. 158-161, 1995.

LOHMAN, T. G.; ROCHE, A.E.; MARTORELL, R. Anthropometric standardization reference manual. Champaign: Human Kinetics, 1988.

PEREIRA, R. A., SICHIERI R.; MARINS, V. M. R. Razão cintura/ quadril como preditor de hipertensão arterial. Caderno de Saúde Pública. v. 15, p. 333-344, 1999.

POWERS, S. K.; HOWLEY, E. T. Fisiologia do exercício: teoria e aplicação ao condicionamento e desempenho. 3. ed. São Paulo: Manole, 2000.

QUEIROGA, M. R. Utilização de medidas antropométricas para a determinação da distribuição de gordura corporal. Revista Atividade Física \& Saúde, Londrina, v. 3, n. 1, p. 37-47,1998

SAMPAIO, L. R.; FIGUEIREDO, V. C. Correlação entre o índice de massa corporal e os indicadores antropométricos de distribuição de gordura corporal em adultos e idosos. Revista de Nutrição v.18, n. 1, p.53-56, 2005.

VALDEZ, R; SEIDELL, J. C; AHN, Y. I; WEISS, K. M. A new index of abdominal adiposity as an indicator of risk for cardiovascular disease. A cross-population study. International Journal of Obesity and Related Metabolic Disorders. v. 17, n. 12, p. 727, 1993

WORLD HEALTH ORGANIZATION. Obesity: preventing and managing the global epidemic. Geneva: World Health Organization; 1998

KANNEL, W. B.; et al. Risk stratification of obesity as a coronary risk factor. American Journal of Cardiology, v. 90 p. 697-701, 2002. 


\section{Romulo Jose Dantas Medeiros}

UFPB

Maria do Socorro Cirilo de Sousa

UFPB

\section{Giulliana Nobrega Cantisani}

UFPB

\section{Anderson Carlos Lourenço de Lima}

Faculdade ASPER

Mônica da Silveira Torres

Universidade da Cidade

João Marcos Ferreira de Lima Silva

UFPB

Luciano Meireles de Pontes,

UFPE,

Luciano Carvalho de Oliveira,

UCB

\section{Gilmario Ricarte Batista}

UFRN

Referência do artigo:

\section{ABNT}

MEDEIROS, J. R. D., et. al. Análise do impacto do icq e cc sobre o risco cardíaco em pessoas com baixos níveis de atividade física. Conexões, v. 6, p. 184-196, 2008.

\section{APA}

Medeiros, R. J. D., Sousa, M. S. C., Cantisani, G. N., Lima, A. C. L., Torres, M. S., Silva, J. M. F. L., Pontes, L. M., Oliveira, L. C., \& Batista, G. R. (2008). Análise do impacto do icq e cc sobre o risco cardíaco em pessoas com baixos níveis de atividade física, 6, 184-196.

\section{VANCOUVER}

Medeiros RJD, Sousa MSC, Cantisani GN, Lima ACL, Torres MS, Silva JMFL, Pontes LM, Oliveira LC, Batista GR. Análise do impacto do icq e cc sobre o risco cardíaco em pessoas com baixos níveis de atividade física.Conexões, 2008; 6: 184-196. 